\title{
Mechanistic insight into electricity generation from moving ionic droplets on graphene
}

\author{
Hongbo Zhang, Zhuhua Zhang ${ }^{*}$ and Wanlin Guo
}

\begin{abstract}
Recent experiments have demonstrated that moving water droplets on polymer-supported graphene can generate electric voltages in graphene. Here, we perform a multi-scale analysis on the mechanism of the generated voltages on the basis of an interplay among the substrate, graphene and ionic water. We find that the attraction of ions in water by substrate dipoles drives charge redistribution in graphene, forming an electric triple layer (ETL) at the water/ graphene/substrate interface, made of an ion layer fixed on graphene, an image charge layer in graphene and a counterion layer in water. As a droplet moves on graphene, dynamic formation of the ETL at its front end drives a flow of charge in graphene. Using Langmuir adsorption theory combined with $a b$ initio calculations, we determine the ion concentration in the ETL and estimate the amount of charge that each ion can draw in graphene. Then, the electric current in graphene is formulated in terms of ion concentration, droplet velocity, graphene thickness and density of substrate dipoles, which well reproduces experimentally measured currents in graphene. These results underscore the importance of tailoring substrate dipoles in optimizing the performance of devices for water energy harvesting and promoting practical applications.
\end{abstract}

Keywords: ion adsorption, drawing potential, atomistic calculation, graphene

\section{INTRODUCTION}

Interfaces between solids and water are ubiquitous in nature and are responsible for many physical phenomena, including wetting on surface [1-3], electrochemical corrosions [4-6] and electrodeposition [7-9]. Thus, the interaction between water and solid surfaces have been extensively studied [10], with aims to identify exotic water structuring at the interface [11-14], achieve high- performance energy storage devices [15-17] and develop new principles for water energy harvesting [18-23]. Water-solid interactions are often dominated by the socalled electric double layer (EDL) formed at solid-liquid interfaces [20,21,24-26], composed of an ion layer firmly adsorbed on the solid surface, called the Stern layer, and a layer of counter-ions in water attracted to the Stern layer, called the diffusion layer. It was found more than a century ago that for water confined in a narrow channel, a relative motion of the diffusion layer to the Stern layer induced by a pressure gradient can yield a potential difference along the channel, referred to as the streaming potential [27]. This electrokinetic effect represents the first form of energy harvesting from water based on a direct solid-water interaction.

In 2014, Yin and cowokers [21] found that drawing ionic droplets or waving ionic water on graphene supported by a polyethylene terephthalate (PET) substrate can generate voltages in graphene proportional to the velocity of water motion. The voltage in graphene induced by drawing droplets has been coined as drawing potential, while that induced by waving water is called waving potential [20]. The drawing and waving potentials are important forms of hydrovoltaic effect [23,28], an emerging field of electricity generation in nanostructured materials on direct interaction with water. The two forms of hydrovoltaic effects share a distinctly different mechanism from that of the streaming potential: the liquidgas interfaces on graphene create boundaries of the EDL between graphene and water, which moves along graphene to generate an electric current in graphene. This mechanism extends the classical electrokinetic theory in light of that it does not require an applied pressure gradient to shear the EDL but relies on a moving boundary

State Key Laboratory of Mechanics and Control of Mechanical Structures, Key Laboratory for Intelligent Nano Materials and Devices of Ministry of Education, and Institute of Nanoscience, Nanjing University of Aeronautics and Astronautics, Nanjing 210016, China

* Corresponding author (email: chuwazhang@nuaa.edu.cn) 
of the EDL [20,23] along functional nanomaterials.

Subsequent experiments have shown that the generated electricity substantially depends on the substrates [2931]. Graphene on a polytetrafluoroethylene (PTFE) substrate has been shown to possess a high carrier density and achieve a 100-fold enhancement of the output power [30]. The drawing potential in graphene on a piezoelectric polyvinylidene fluoride substrate can reach $0.1 \mathrm{~V}$ even without salt in water [29]. These results suggest an important interaction of the surface dipoles of substrates with the EDL formed between graphene and water, which dominates the output power in graphene. This interaction was further revealed by Yang et al. [31] via sum-frequency vibrational spectroscopy. By comparing the ion distributions at water/graphene interfaces on PET with surface dipoles and those on polymethyl methacrylate (PMMA) without the dipoles, they concluded that the surface dipole layer is responsible for selective ion adsorption on graphene, while graphene itself acts as a conducting sheet to transport induced carriers [32,33]. This study opens a new prospect to enhance the device performance by optimizing the substrates. Later on, droplet energy harvesting was generalized to more systems, including a droplet falling into an electrolyte solution [34] and a droplet hitting onto a charged polymer substrate [19]. Despite a plenty of experimental studies [19,29-31,34-36] following the waving and drawing potentials, a quantitative theoretical analysis on their mechanism has yet to be provided, as hampered by complicated multiscale processes spanning from ionelectron interaction at microscale to the measured electric power at macroscale.

In this work, we carry out a multiscale study of the electricity generation by moving an ionic droplet on PETsupported graphene using combined Langmuir adsorption theory [37-39], ab initio calculations and electrostatic simulations. We consider an image charge layer in graphene, which couples with the EDL formed between graphene and water to constitute an electric triple layer (ETL). As the droplet moves forwards on graphene, the ETL at the front end is on the way to an equilibrium, in which each cation attracted towards graphene draws a certain amount of electrons from the unwetted part of graphene. On the basis of this picture, we formulate the electric current generated in graphene as a function of several key physical parameters of the water/graphene/ substrate system, and achieve a reasonable agreement with experimental measurements. Our analysis suggests that the electric power can be greatly enhanced by increasing the moment of surface dipoles of substrates, but reduced by densifying the surface dipoles relative to those on the PET.

\section{RESULTS}

\section{Models}

Our model is composed of $\mathrm{NaCl}$ aqueous solution, graphene and a PET substrate (Fig. 1a). We refered to the $\mathrm{NaCl}$ aqueous solution as water unless specially specified. On the PET, the surface dipoles were manifested as periodically arranged carbonyl groups $(\mathrm{C}=\mathrm{O})$ with negatively charged $\mathrm{O}$ atoms pointing outward the substrate surface [40]. Since graphene is only one-atom thick, it cannot fully screen the electric field from the $\mathrm{O}$ atoms (or dipoles). As such, the $\mathrm{Na}^{+}$ions in water were attracted towards graphene by the surface dipoles, driving the formation of an EDL between water and graphene [31]. The graphene itself then acted as a conducting sheet to adapt the interaction between the $\mathrm{Na}^{+}$and the dipoles. As has been revealed by Yin et al. [20], the movement of EDL boundary is vital to the electricity generation, which we analyzed in details in this work.

\section{Moving boundaries of ETL}

In the region of an EDL, the rates of adsorption and desorption reactions have reached a dynamic equilibrium, under which we treated the EDL as a static system. Once the EDL boundary moves forward, the EDL is forming at the front edge, with a lower density of adsorbed $\mathrm{Na}^{+}$than

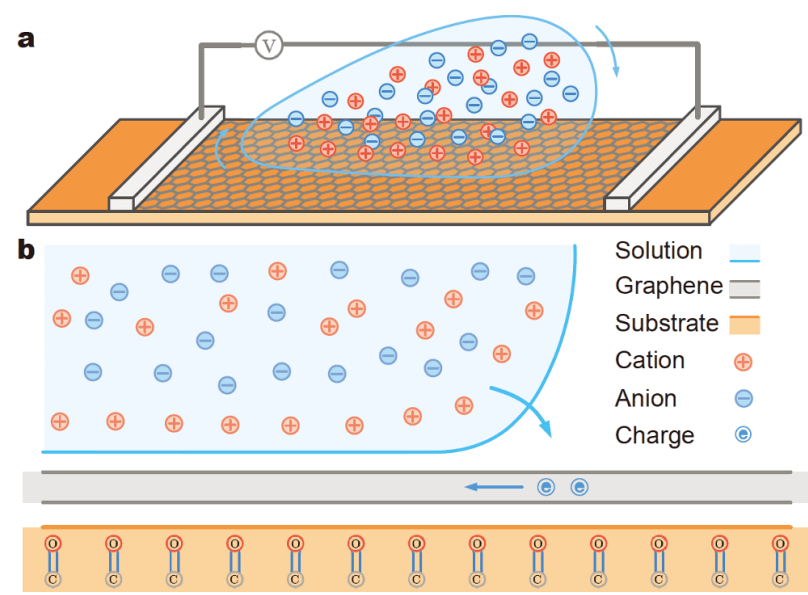

Figure 1 A schematic model for the drawing potential. (a) When a water droplet moves forward on a PET-supported graphene, the ions are adsorbed on the graphene and an ETL forms between graphene and water. (b) Cations are attracted by negative $\mathrm{O}$ atoms in $\mathrm{C}=\mathrm{O}$ groups on the PET substrate; near the front end of the droplet, new ETL consistently forms upon the droplet motion, dragging electrons in graphene (image charge layer) nearby flowing backward. 
that in the equilibrated regions. Thus, the difference in ion density causes a local potential gradient driving electrons in graphene to move reversely. The dynamic image charge layer in graphene, together with the EDL, forms an ETL as shown in Fig. 1b. As it takes only several nanoseconds for the adsorption reaction to reach equilibrium, the potential gradient only spans several nanometers in the moving direction. We used a simple model to delineate this complex process: when the ETL boundary moves across a given adsorption site, a $\mathrm{Na}^{+}$ attracted towards the site draws a reverse flow of electrons in the image charge layer. The density of adsorption sites equals the equilibrium density of the adsorbed sodium ions. With this model, we formulated the generated current using a multiscale method. We separated the procedure of formulation into two steps. First, we used the traditional adsorption theory to describe how many cations can be adsorbed on the graphene. Second, we determined how electrons were redistributed in graphene upon the adsorption of ions on sites by employing density functional theory (DFT) calculations; we then determined how many electrons each adsorbed $\mathrm{Na}^{+}$can draw.

\section{Traditional adsorption theory}

We started the first step by resorting to the traditional adsorption theory. $\mathrm{Na}^{+}$ions in water are adsorbed on graphene by periodically arranged surface dipoles on the substrate, via electrostatic interaction across the graphene layer. The surface dipoles on the PET are in the form of $\mathrm{C}=\mathrm{O}$ groups with a dipole moment of $\sim 2.7 \mathrm{D}(\delta=0.47 e, l$ $=1.2 \AA)$. While the dipoles are quite strong compared with other functional groups, they do not suffice to cause multilayer adsorption of cations on graphene. This feature allows us to use the Langmuir adsorption model, according to which the relationship among the surface coverage $\Theta$ of cations, the ion concentration and the equilibrium constant $K$ is expressed as:

$\Theta=\frac{K \cdot c}{1+K \cdot c}$,

where $c$ is the ion concentration and $K=k_{\text {ad }} / k_{\text {des. }}$. Here, $k_{\mathrm{ad}}$ is the reaction rate constant for the adsorption rate $R_{\mathrm{ad}}$, while $k_{\text {des }}$ is that for the desorption rate $R_{\text {des }} . R_{\text {ad }}$ and $R_{\text {des }}$ can be expressed as:

$R_{\text {ad }}=k_{\text {ad }}(T) c S_{0}$,

$R_{\mathrm{des}}=k_{\mathrm{des}}(T) S_{1}$,

where $S_{0}$ is the fraction of uncovered surface (proportional to $1-\Theta$ ), and $S_{1}$ is the fraction of covered surface (proportional to $\Theta$ ). Then, we derived the specific expressions of adsorption and desorption rates, $R_{\mathrm{ad}}$ and $R_{\mathrm{des}}$.

\section{Rate of adsorption}

According to the simple collision theory, reaction rate constant is the product of collision frequency (or frequency factor, pre-exponential factor) and fraction of effective collision. Thus, the adsorption rate can be related to the collision frequency of ions with an adsorbent wall (i.e., graphene), and the collision efficiency. The averaged moving velocity of a hydrated $\mathrm{Na}^{+}$at a temperature $T$ is written as:

$\bar{v}=\sqrt{\frac{8 k T}{\pi m}}$,

where $k$ is the Boltzmann constant and $m$ is the mass of a hydrated sodium ion, $\sim 131 \mathrm{u}$. The probability for the hydrated $\mathrm{Na}^{+}$to collide with the adsorbent wall is calculated to be 1/4 (see section S1, Fig. S1 in Supplementary information (SI) for details). The collision frequency is written as:

$\frac{\mathrm{d} N}{A \cdot \mathrm{d} t}=\frac{1}{4} c N_{\mathrm{A}} \bar{v}$,

where $N_{\mathrm{A}}$ is the Avogadro constant. We considered the collision of a hydrated $\mathrm{Na}^{+}$with an adsorbent wall to be efficient only when the ion hits the plane within an efficient area and its kinetic energy is lower than the adsorption energy $E_{\text {ad }}$. Here, the efficient area is a circle, with a radius $r$ and a center at the adsorption site. If we took saturated ion density $\sigma_{\text {sat }}$ as the density of adsorption sites, the probability of hitting the efficient area will be $P_{\text {hit }}=\pi r^{2} \sigma_{\text {sat. }}$. For the PET, $\sigma_{\text {sat }}$ can be approximated as the density $\left(0.235 \mathrm{~nm}^{-2}\right)$ of the $\mathrm{C}=\mathrm{O}$ groups on the substrate [31].

Then, the kinetic energy of hydrated ions obeys the Maxwell-Boltzmann energy distribution as:

$f\left(E_{\mathrm{k}}\right)=\frac{2}{\sqrt{\pi}}(k T)^{-3 / 2} E_{\mathrm{k}}^{1 / 2} e^{-\frac{E_{\mathrm{k}}}{k T}}$.

By integrating the Maxwell-Boltzmann energy distribution, we can obtain the fraction of ions whose kinetic energy $E_{\mathrm{k}}$ is lower than $E_{\mathrm{ad}}$ :

$P_{E_{\mathrm{k}}<E_{\mathrm{ad}}}=\int_{0}^{E_{\mathrm{ad}}} f\left(E_{\mathrm{k}}\right) \mathrm{d} E_{\mathrm{k}}$.

Then, the adsorption rate can be expressed as:

$$
\begin{aligned}
R_{\mathrm{ad}} & =\frac{P_{\mathrm{hit}} \mathrm{d} N}{A \cdot \mathrm{d} t} P_{E_{\mathrm{k}}<E_{\mathrm{ad}}} S_{0} \\
& =\frac{1}{4} \cdot c N_{\mathrm{A}} \bar{v} \cdot \pi r^{2} \sigma_{\mathrm{sat}} \cdot P_{E_{\mathrm{k}}<E_{\mathrm{ad}}} \cdot S_{0} .
\end{aligned}
$$

\section{Rate of desorption}

The desorption rate of ions can be expressed as a product of attempt frequency of desorption and fraction of effective attempt. We described the profile of the potential 
well induced by each surface dipole as a conic section (see section S2 in SI), so that each adsorbed ion experiences a simple harmonic vibration in the well. As such, we can calculate the attempt frequency from the vibrating frequency. With a vibration amplitude $A$ and a desorption energy $E_{\mathrm{des}}$, the vibrating frequency $f$ can be calculated as:

$f=\frac{1}{2 \pi A} \sqrt{\frac{2 E_{\mathrm{des}}}{m}}$.

As shown in Fig. S2c, the profile of the potential well is wide enough to consider every desorbing attempt with ion's kinetic energy exceeding $E_{\text {des }}$ as effective. Then we formulated the desorption rate as:

$$
\begin{aligned}
R_{\mathrm{des}} & =f P_{E_{\mathrm{K}}>E_{\mathrm{des}}} S_{1} \\
& =\frac{1}{2 \pi A} \sqrt{\frac{2 E_{\mathrm{des}}}{m}} \cdot P_{E_{\mathrm{K}}>E_{\mathrm{des}}} \cdot S_{1} .
\end{aligned}
$$

As the adsorption of ions on graphene is dominated by electrostatic interaction, we considered $E_{\text {des }}=E_{\text {ad }}$.

\section{Equilibrium constant}

The equilibrium constant of adsorption reaction (i.e., $k_{\mathrm{ad}} /$ $k_{\text {des }}$ ) is the reaction quotient under dynamic equilibrium. A larger equilibrium constant results in a higher density of adsorbed ions. With these provisions, we can use Equations (8 and 10) to calculate the adsorption and desorption rates, use Equations ( 2 and 3 ) to derive $k_{\text {ad }} /$ $k_{\text {des }}$, and use Equation (1) to obtain the equilibrated surface coverage $\Theta$. Finally, we calculated the density of adsorbed ions as:

$\sigma=\sigma_{\text {sat }} \Theta=\sigma_{\text {sat }} \frac{K c}{1+K c}$.

We set $2 r=10.0 \AA$ as the distance between two adjacent dipoles on the substrate, $A=3.5 \AA$ and $E_{\text {ad }}=0.09 \mathrm{eV}$ according to the adsorption energy profile calculated by DFT (Fig. S3b). Then, we obtain $\sigma$ as a function of $c$, as shown in Fig. 2a, which agrees well with experiment data.
For example, at $c=0.6 \mathrm{~mol} \mathrm{~L}^{-1}$, the calculated density of adsorbed ions is $0.069 \mathrm{~nm}^{-2}$, comparable to an experimental value of $0.065 \pm 0.011 \mathrm{~nm}^{-2}$.

\section{Relaxation time}

The charge relaxation time of an EDL (equals that of an ETL since electrons move much faster than ions) can be estimated as $\tau_{\mathrm{D}}=\lambda_{\mathrm{D}}{ }^{2} / D$, where $\lambda_{\mathrm{D}}$ is the Debye screening length and $D$ is the diffusivity of ions. For an ion concentration of $c=0.6 \mathrm{~mol} \mathrm{~L}^{-1}, \lambda_{\mathrm{D}}=12.6 \mathrm{~nm}$ and $D=$ $0.17 \mu \mathrm{m} \mathrm{s}^{-1}$, which is the average of the diffusivities of $\mathrm{Na}^{+}\left(0.13 \mu \mathrm{m} \mathrm{s}^{-1}\right)$ and $\mathrm{Cl}^{-}\left(0.2 \mu \mathrm{m} \mathrm{s}^{-1}\right)$, and then $\tau_{\mathrm{D}}$ in the ETL was calculated to be 0.93 ns. To verify our theory, we employed Equations (8 and 10) to determine the relaxation time. According to the reaction kinetics of adsorption, the relationship among $\Theta, k_{\text {ad }}$ and $k_{\text {des }}$ is expressed as:

$\dot{\Theta}+\left(k_{\mathrm{ad}}+k_{\mathrm{des}}\right) \Theta-k_{\mathrm{ad}}=0$,

which is a differential equation. By applying the boundary conditions $\left.\Theta\right|_{t=0}=0$ and $\left.\Theta\right|_{t=\infty}=k_{\text {ad }} /\left(k_{\text {ad }}+k_{\text {des }}\right)=\Theta_{\text {eq }}$ (i.e., the equilibrium surface coverage), we obtained the solution as:

$\Theta=\Theta_{\mathrm{eq}}\left(1-\exp \left(-\left(k_{\mathrm{ad}}+k_{\mathrm{des}}\right) t\right)\right)$.

We defined the relaxation time as the time needed for $\Theta$ reaching $95 \%$ of $\Theta_{\text {eq }}$. With Equation (13), we estimated the relaxation time to be $0.4 \mathrm{~ns}$ (Fig. $2 \mathrm{~b}$ ), which is in a same order of magnitude with $0.93 \mathrm{~ns}$ set forth above. This agreement supports the reliability of the calculated adsorption and desorption rates.

\section{Electron redistribution in graphene}

We next discussed the second step by employing DFT calculations to determine the electron redistribution in graphene. To capture the essential physics, we simplified the model as PET-supported graphene on which a hy-
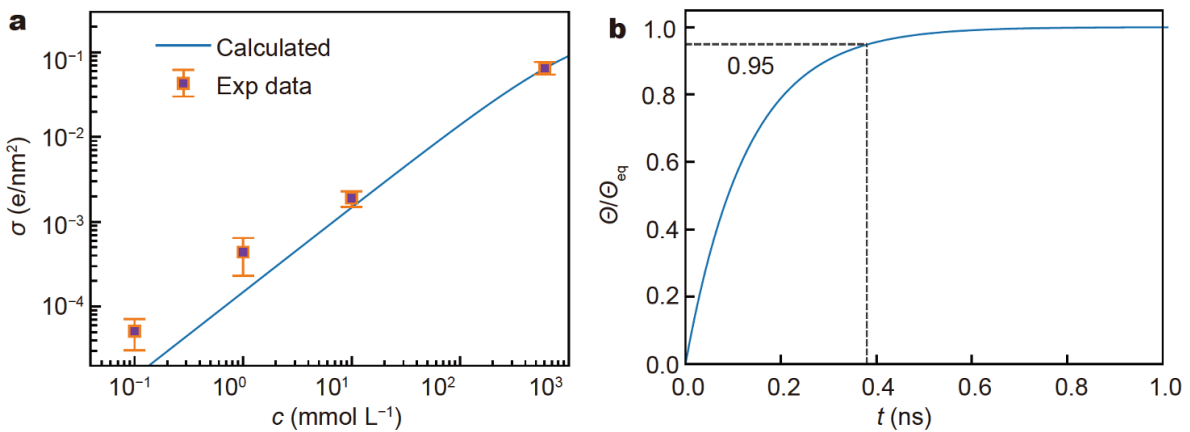

Figure 2 Results calculated by the traditional adsorption theory. (a) Calculated density $\sigma$ of the adsorbed ions on graphene (line), compared with the experimental results (purple squares) of Yang et al. [31]. (b) Evolution of relative surface coverage of the adsorbed ions, $\Theta / \Theta_{\text {eq, }}$, on graphene with time. The system takes about $0.4 \mathrm{~ns}$ to reach equilibrium $\left(\Theta / \Theta_{\mathrm{eq}}=95 \%\right.$, purple cross $)$. 
drated ion is adsorbed. In reality, PET is composed of polymer high-molecules, each with thousands of atoms, but the critical motifs responsible for the ion adsorption are the polar $\mathrm{C}=\mathrm{O}$ groups. We used a piece of molecule $\mathrm{CO}\left(\mathrm{CH}_{3}\right)_{2}$ to include the effect of a $\mathrm{C}=\mathrm{O}$ group in our models, along with a orthogonal supercell with a lateral dimension of $21.4 \times 19.7 \AA^{2}$ based on previously estimated density of $\mathrm{C}=\mathrm{O}$ groups on PET [31]. Note that the periodical boundary condition leads to a condition that the surface dipoles are evenly distributed on the PET. Yet, the practical inhomogeneity of surface dipole density is expected to have little effect on our results (see S5 section in $\mathrm{SI}$ ). The adsorbed ion is surrounded by five water molecules for hydration. We relaxed the whole system and evaluated the electron redistribution in graphene upon the ion adsorption.

At the equilibrium state, the sodium ion sits at $3.56 \AA$ over the graphene layer, while $\mathrm{O}$ atom of the $\mathrm{C}=\mathrm{O}$ group is $3.11 \AA$ below the layer. Fig. 3a displays that, upon the adsorption of a $\mathrm{Na}^{+}$, electrons transfer from the surrounding clean area of graphene to that right below the adsorbed ion. The distribution of transferred electrons exhibits an apparent symmetry respecting to the normal axis going through the $\mathrm{Na}^{+}$. With this normal axis, we defined a cylinder to cover the regions of the accumulated electrons (Fig. 3a). The radius of the cylinder is taken as the distance between the axis and a place where the local electron distribution is nearly zero. Combining this model with DFT results, we found that an adsorbed sodium ion can drag $\Delta q \approx 0.22 e$ from the surrounding graphene area (image charge layer in ETL).

Since direct DFT calculations of $\Delta q$ at different densities of the adsorbed ions and substrate dipoles are rather cumbersome, we turned to achieve an analytical expression of $\Delta q$. In view of the cylindrical distribution of $\Delta q$, we integrated the transferred electrons along the circumference and then summarized all those transferred electrons onto a radius-defined rectangle, as illustrated in Fig. 3c. Note that electrons transferred from the underside of graphene to its upper side do not contribute to $\Delta q$, and are thus neglected here.

The electron transfer in graphene induced by an ex-
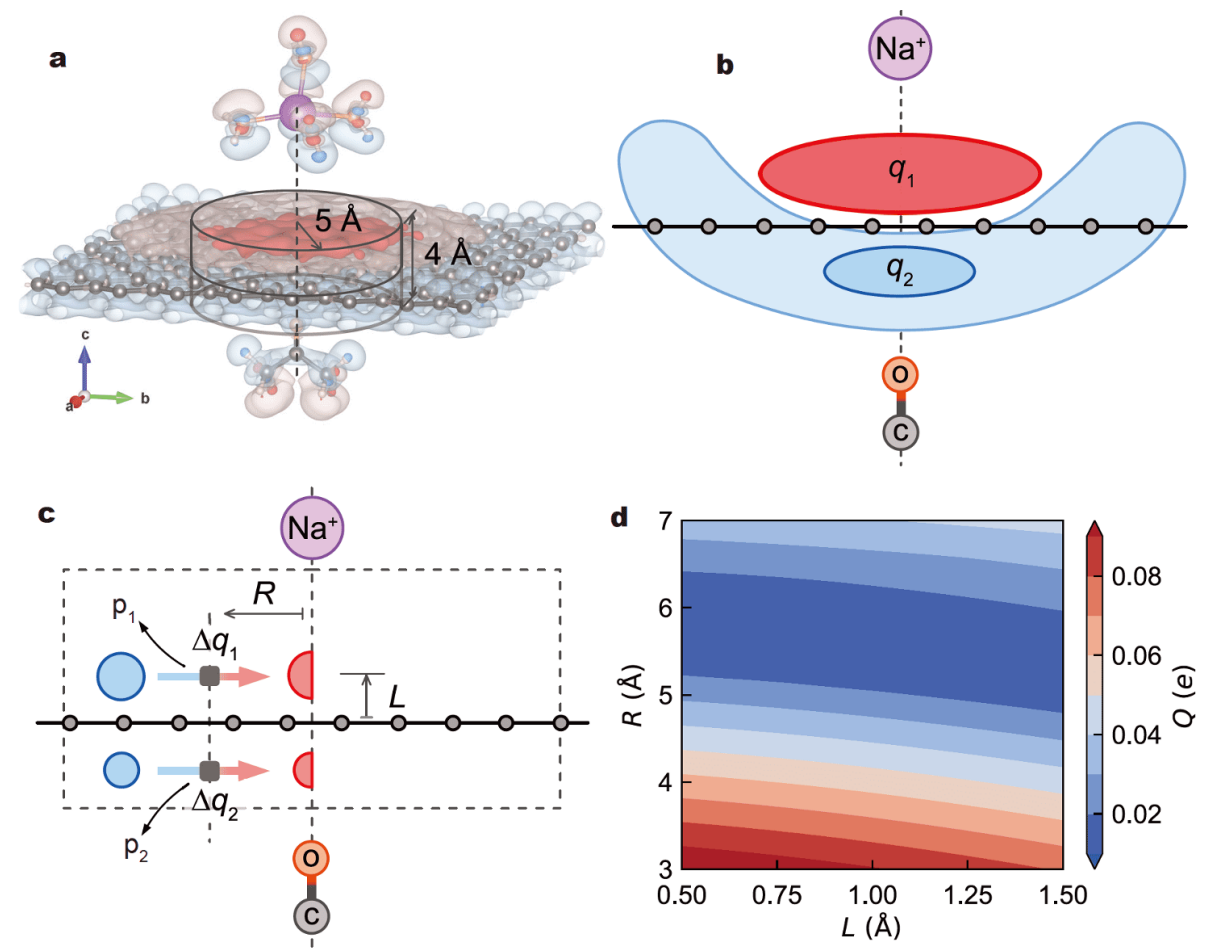

Figure 3 Electron redistribution in graphene induced by an adsorbed $\mathrm{Na}^{+}$. (a) Iso-surface plot $\left(0.001,0.005 \mathrm{e} / \AA^{3}\right)$ of charge density difference. The electrons transfer from the blue region to the pink region. The cylinder represents the space within which we determine the transferred electrons upon the adsorption of $\mathrm{Na}^{+}$. (b) A schematic illustration of the electron transfer in graphene. (c) Our model for determining the transferred electrons into the cylinder shown in (a). Here, the transferred electrons from all directions into the cylinder sections below and above the graphene plane are summarized as $\Delta q_{1}$ and $\Delta q_{2}$, respectively. The two points $\mathrm{p}_{1}$ and $\mathrm{p}_{2}$ are the position where we calculate the external field. (d) Distribution of the difference between $\Delta q$ determined with the model and DFT in the parameter space of $R$ and $L$ with single-layer graphene (see definitions in (c)). $Q$ is defined in the text. For the situation where $R=5.0 \AA$ and $L=1.0 \AA, Q=0.021 \mathrm{e}$. 
ternal field $E_{\text {ext }}$ can generate an internal field $E_{\text {int }}$ in an opposite direction. According to electrostatics in dielectric materials, the relation between $E_{\text {int }}$ and $E_{\text {ext }}$ is written as:

$E_{\text {int }}=\frac{1-\varepsilon_{\mathrm{r}}}{\varepsilon_{\mathrm{r}}} E_{\text {ext }}$,

where $E_{\text {ext }}$ is generated by the $\mathrm{Na}^{+}$and the $\mathrm{C}=\mathrm{O}$ group, and $\varepsilon_{\mathrm{r}}$ is the (relative) dielectric constant of the material. The transferred charge can be divided into two parts, the one above the graphene layer $\left(\Delta q_{1}\right)$ and the other below the layer $\left(\Delta q_{2}\right) . \Delta q_{1}$ and $\Delta q_{2}$ can induce two internal fields. We assumed that the distance of electron transfer is $2 R$, and $\Delta q_{1}$ and $\Delta q_{2}$ are away from the graphene layer by $L$. Then, $E_{\text {int }}$ has an expression:

$E_{\text {int }}^{i}=\frac{1}{2 \pi \varepsilon_{0} \varepsilon_{\mathrm{r}}} \frac{\Delta q_{i}}{R^{2}}$,

where $i=1,2$, and $\varepsilon_{0}$ is the dielectric constant in free space. By combining Equations (14 and 15), we wrote $\Delta q_{1}$ and $\Delta q_{2}$ as (Fig. 3 c):

$\Delta q_{i}=\frac{1-\varepsilon_{\mathrm{r}}}{\varepsilon_{\mathrm{r}}} 2 \pi \varepsilon_{0} \varepsilon_{\mathrm{r}} R^{2} E_{\mathrm{ext}}^{i}$,

where $i=1,2$. The total amount of transferred electrons is

$\Delta q=\Delta q_{1}+\Delta q_{2}$.

The dielectric constant of graphene has been determined by Santos and cowokers [41] using DFT calculations. They found that both out-of-plane $\left(\varepsilon_{\perp}\right)$ and inplane $\left(\varepsilon_{\|}\right)$relative dielectric constants depend on the applied electric field and the size of graphene. Based on their results, we took $\varepsilon_{\perp} \approx 3$ and $\varepsilon_{\|} \approx 1.8$.

Finally, we can calculate $E_{\text {ext }}{ }^{1}$ at the site of $\Delta q_{1}$ and $E_{\text {ext }}{ }^{2}$ at the site of $\Delta q_{2}$ (point $\mathrm{p}_{1}$ and $\mathrm{p}_{2}$ in Fig. 3c) as functions of $R$ and $L$, which enable us to easily determine $\Delta q$ using Equations (16 and 17). From the DFT-calculated profile of electron transfer shown in Fig. 3a, we can measure $R=$ $5 \AA$ and $L=1 \AA$; then our analysis yields $\Delta q=0.19 e$, close to $0.22 e$ from direct DFT calculations.

It is necessary to examine the suitability of the parameters $R$ and $L$. For this purpose, we calculated $\Delta q$ as a function of the distance between the $\mathrm{Na}^{+}$and graphene with DFT, comparable to the corresponding values $\Delta q^{\prime}$ obtained from our model. The difference between them is denoted by $Q$, and

$Q=\operatorname{mean}\left(\Delta q-\Delta q^{\prime}\right)$.

The results in Fig. $3 \mathrm{~d}$ indicate that the $Q$ does not change substantially with $L$ in the region around $1 \AA$, but will decrease from 0.021 e at $R=5.0 \AA$ to $0.012 \mathrm{e}$ at $R=$ $5.5 \AA$ (minimal) with $L=1 \AA$. Note that $R=5 \AA$ is just one quarter of the distance between two neighboring $\mathrm{C}=\mathrm{O}$ groups. As the electron redistribution is too rambling to make a feasible counting of transferred electrons when $\mathrm{Na}^{+}$is near the graphene layer (Fig. S4), the transferred electrons counted from DFT calculation is not reliable under this circumstance. Therefore, we used this $R$ (one quarter of the distance between two neighboring $\mathrm{C}=\mathrm{O}$ groups) rather than $\sim 5.5 \AA$ appeared as a valley in Fig. $3 \mathrm{~d}$ for following computations with different densities of surface dipoles.

\section{Computational results}

Having discussed the two steps of formulation, we proceeded by deriving the analytical form of the generated current in graphene by moving a droplet on it. The current $I$ is defined as the amount of charge $\Delta Q$ flowing through a cross section of graphene (i.e., a line due to atomic thickness) in a given time $\Delta t$. For a droplet with a diameter $d$ and a moving velocity $v, \Delta Q$ can be expressed as:

$\Delta Q=d \cdot v \Delta t \cdot \sigma \Delta q$.

Then, $I$ can be expressed as:

$I=\frac{\mathrm{d} Q}{\mathrm{~d} t}=\frac{d \cdot v \Delta t \cdot \sigma \Delta q}{\Delta t}=d v \sigma \Delta q$,

where $\sigma$ and $\Delta q$ can be calculated with Equations (11 and 17), respectively.

According to the experiment of the drawing potential by Yang et al. [31], $d \approx 5 \mathrm{~mm}, c=0.6 \mathrm{~mol} \mathrm{~L}^{-1}, v \approx 1 \mathrm{~m} \mathrm{~s}^{-1}$, with which we calculated the current in a single-layer graphene to be $I=9.9 \mu \mathrm{A}$, close to their measured current of $9.1 \mu \mathrm{A}$. This good agreement between the theory and experiment further validates our model and the key physical mechanism regarding the electricity generation in graphene by moving a droplet over it.

The number of graphene layers, the density of surface dipoles and the moment of dipoles are three important parameters governing the generated current. We calculated the current as a function of these parameters separately (Fig. 4). The current decreases dramatically with increasing number of graphene layers, from $9.9 \mu \mathrm{A}$ in a single-layer graphene, $3.8 \mu \mathrm{A}$ in a bilayer graphene, to $1.6 \mu \mathrm{A}$ in a tri-layer graphene. This is attributed to a decrease of the density of adsorbed ions on graphene as a result of weakened interaction between the ions and the substrate dipoles. According to the measured resistances of single-layer, bilayer and tri-layer graphene and sizes of employed samples from the experiment [20], we determined the generated voltage to be $2.8 \mathrm{mV}$ in a singlelayer graphene as measured. Our analysis shows that the 

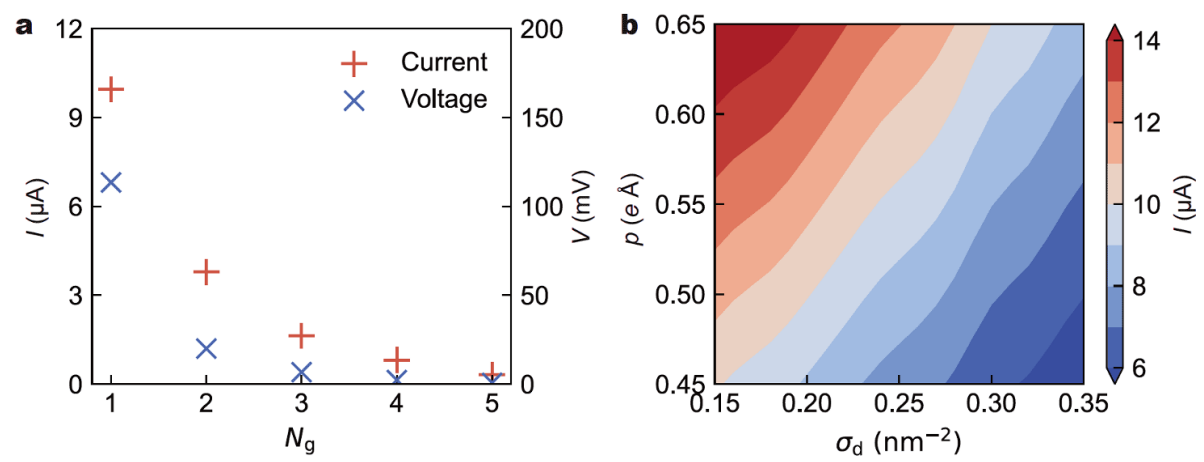

Figure 4 The computation results. (a) Calculated current $I$ and voltage $V$ as functions of the number of graphene layer $N_{\mathrm{g}}$. (b) A contour plot of the calculated current $I$ in the parameter space of the density of surface dipoles $\sigma_{\mathrm{d}}$ and the moment of dipoles $p$.

voltage drops to $0.43 \mathrm{mV}$ in a bilayer graphene and only $0.17 \mathrm{mV}$ in a tri-layer graphene. While these values cannot be directly compared with the experimental values by Yin et al. [20] due to the difference in samples and style of water motion, $85 \%$ decrease of the generated voltage in the bilayer graphene and $94 \%$ decrease in the trilayer graphene with respect to that in the single-layer agree well with experimentally measured variation trend. Such a sharp decrease of voltage results from the decline of both the generated current and graphene resistance.

The results show that intensifying the substrate dipoles raises the electric current, due to the enhanced ion-dipole attraction. For example, at a given dipole density of $0.25 \mathrm{~nm}^{-2}$, the current increases from $8.6 \mu \mathrm{A}$ at a moment of $0.50 e \AA$ to $10.8 \mu \mathrm{A}$ at a moment of $0.60 e \AA$. In contrast, graphene on a substrate with a moderately higher dipole density tends to generate a lower current, as a result of the reduced $\Delta q$ dragged by each adsorbed ion. With a moment of substrate dipoles of $0.55 e \AA$, the current generated decreases from $11.3 \mu \mathrm{A}$ at a dipole density of $0.20 \mathrm{~nm}^{-2}$ to $8.0 \mu \mathrm{A}$ at a dipole density of $0.30 \mathrm{~nm}^{-2}$.

\section{DISCUSSION}

We have adopted several assumptions for deriving the generated current. First, in the section of traditional adsorption theory, we used $r$, the radius defining an effective collision area in the graphene wall for an ion, to calculate the adsorption rate, and used $A$, the oscillation magnitude of an adsorbed ion, to calculate the desorption rate. Determining $r$ and $A$ is crucial to final results and in our theory is based on COMSOL and DFT calculations, respectively. More precise methods are expected to calibrate their values. Second, we only consider a single-layer of adsorbed ions, and neglect the interaction between ad- sorbed ions. For the case with a high salt concentration and a large moment of the substrate dipoles, a multilayer of adsorbed ions may arise near the interface and should be considered for reliably quantifying the adsorbed ions on graphene. For the case with a density of surface dipoles one hundred times higher than those on the PET, the density of surface dipoles cannot be taken as the density of saturated ions $\sigma_{\text {sat }}$, due to greatly enhanced ionion interaction that makes a part of sites deprived of adsorbing ions.

Recently, Xu et al. [19] found that dropping a droplet onto a PTFE film supported by an indium tin oxide and overlaid by a piece of aluminum electrode, can generate an instant voltage of up to $100 \mathrm{~V}$, significantly higher than the values of drawing potential [21]. The origin of the high instant voltages has been analytically related to the evolution of the drop-substrate interfacial area $[18,42]$, but its atomistic mechanism remains to be explored. The proposed model in this work may serve as a step-stone towards understanding the energy harvesting from falling onto a pre-charged surface. In our model, the moving boundary of an ETL drives an electric current in graphene, whereas the distinct voltages in the work of $\mathrm{Xu}$ and coworkers [19] arise only when the drop's edge is connecting the aluminum electrode. We speculate that the aluminum electrode plays a role in shearing the EDL formed between the drop and the PTFE film and thereby efficiently extracts the electric energy stored in the EDL. Fully considering the dynamic evolution of the EDL and its new dynamic extension near the drop-electrode interface may enable a deep insight into this intriguing phenomenon, which will be a topic of future study. The results based on the water-conductor interaction should be extendable to other monolayer metallic materials, such as borophene [43]. 


\section{CONCLUSION}

We have performed an in-depth multi-scale analysis of the mechanism on electricity generation from moving ionic droplets along PET-supported graphene by combining the traditional adsorption theory, classical electrostatics and DFT calculations. We formulate the generated current in terms of ion concentration, graphene thickness as well as density and moment of surface dipoles on the substrate. The analytical results agree with experimental measurements on graphene of different thicknesses. In particular, we find that moderately densifying the substrate dipoles relative to those on the PET cannot boost the output power. In contrast, intensifying the surface dipoles or decreasing the thickness of graphene layer can effectively raise the current. These results not only deepen the understanding of water energy harvesting based on solid-water interaction, but also will inform on-going efforts of optimizing the performance of hydrovoltaic devices.

Received 1 December 2020; accepted 8 January 2021; published online 29 March 2021

1 Henderson M. The interaction of water with solid surfaces: Fundamental aspects revisited. Surf Sci Rep, 2002, 46: 1-308

2 Zheng JM, Chin WC, Khijniak E, et al. Surfaces and interfacial water: Evidence that hydrophilic surfaces have long-range impact. Adv Colloid Interface Sci, 2006, 127: 19-27

3 Chen P, Chen L, Han D, et al. Wetting behavior at micro-/ nanoscales: Direct imaging of a microscopic water/air/solid threephase interface. Small, 2009, 5: 908-912

4 Yadav DK, Chauhan DS, Ahamad I, et al. Electrochemical behavior of steel/acid interface: Adsorption and inhibition effect of oligomeric aniline. RSC Adv, 2013, 3: 632-646

5 Stansbury EE, Buchanan RA. Fundamentals of Electrochemical Corrosion. Ohio: ASM international, 2000

6 Guillaumin V, Schmutz P, Frankel GS. Characterization of corrosion interfaces by the scanning Kelvin probe force microscopy technique. J Electrochem Soc, 2001, 148: B163

7 Endres F, Abbott A, MacFarlane DR. Electrodeposition from Ionic Liquids. Weinheim: John Wiley \& Sons, 2017

8 Su YZ, Fu YC, Wei YM, et al. The electrode/ionic liquid interface: Electric double layer and metal electrodeposition. ChemPhysChem, 2010, 11: 2764-2778

9 Zheng J, Zhao Q, Tang T, et al. Reversible epitaxial electrodeposition of metals in battery anodes. Science, 2019, 366: 645-648

10 Leng J, Guo Z, Zhang H, et al. Negative thermophoresis in concentric carbon nanotube nanodevices. Nano Lett, 2016, 16: 63966402

11 Xu Y, Xuan X, Zhang Z, et al. A helical monolayer ice. J Phys Chem Lett, 2020, 11: 3860-3865

12 Kim J, Opdahl A, Chou KC, et al. Hydrophobic-interactioninduced alignment of polymers at the solid/liquid interface studied by infrared-visible sum frequency generation. Langmuir, 2003, 19: 9551-9553

$13 \mathrm{Li} \mathrm{CY}$, Le JB, Wang YH, et al. In situ probing electrified interfacial water structures at atomically flat surfaces. Nat Mater, 2019, 18: 697-701

14 Xu Y, Xuan X, Zhang Z, et al. A folded ice monolayer. Phys Chem Chem Phys, 2020, 22: 20388-20393

15 Banerjee S, Han X, Thoi VS. Modulating the electrode-electrolyte interface with cationic surfactants in carbon dioxide reduction. ACS Catal, 2019, 9: 5631-5637

16 Zhang $\mathrm{H}$, Jeong S, Qin B, et al. Towards high-performance aqueous sodium-ion batteries: Stabilizing the solid/liquid interface for NASICON-type $\mathrm{Na}_{2} \mathrm{VTi}\left(\mathrm{PO}_{4}\right)_{3}$ using concentrated electrolytes. ChemSusChem, 2018, 11: 1382-1389

17 Lei Y, Han D, Dong J, et al. Unveiling the influence of electrode/ electrolyte interface on the capacity fading for typical graphitebased potassium-ion batteries. Energy Storage Mater, 2020, 24: 319-328

$18 \mathrm{Wu} \mathrm{H}$, Mendel N, van den Ende D, et al. Energy harvesting from drops impacting onto charged surfaces. Phys Rev Lett, 2020, 125: 078301

$19 \mathrm{Xu} \mathrm{W}$, Zheng H, Liu Y, et al. A droplet-based electricity generator with high instantaneous power density. Nature, 2020, 578: 392-396

20 Yin J, Zhang Z, Li X, et al. Waving potential in graphene. Nat Commun, 2014, 5: 3582

21 Yin J, Li X, Yu J, et al. Generating electricity by moving a droplet of ionic liquid along graphene. Nat Nanotech, 2014, 9: 378-383

22 Yin J, Zhang Z, Li X, et al. Harvesting energy from water flow over graphene? Nano Lett, 2012, 12: 1736-1741

23 Zhang Z, Li X, Yin J, et al. Emerging hydrovoltaic technology. Nat Nanotech, 2018, 13: 1109-1119

24 Grahame DC. The electrical double layer and the theory of electrocapillarity.. Chem Rev, 1947, 41: 441-501

25 Carnie SL, Torrie GM. The statistical mechanics of the electrical double layer. Adv Chem Phys, 1984, 56: 141-253

26 Block LP. A double layer review. Astrophys Space Sci, 1978, 55: 5983

27 Stone HA, Stroock AD, Ajdari A. Engineering flows in small devices. Annu Rev Fluid Mech, 2004, 36: 381-411

28 Yin J, Zhou J, Fang S, et al. Hydrovoltaic energy on the way. Joule, 2020, 4: 1852-1855

29 Zhong H, Xia J, Wang F, et al. Graphene-piezoelectric material heterostructure for harvesting energy from water flow. Adv Funct Mater, 2017, 27: 1604226

30 Kwak SS, Lin S, Lee JH, et al. Triboelectrification-induced large electric power generation from a single moving droplet on graphene/polytetrafluoroethylene. ACS Nano, 2016, 10: 7297-7302

31 Yang $\mathrm{S}, \mathrm{Su} \mathrm{Y}, \mathrm{Xu} \mathrm{Y}$, et al. Mechanism of electric power generation from ionic droplet motion on polymer supported graphene. J Am Chem Soc, 2018, 140: 13746-13752

32 Wei $\mathrm{N}$, Chen $\mathrm{Y}$, Cai K, et al. Thermal conductivity of graphene kirigami: Ultralow and strain robustness. Carbon, 2016, 104: 203 213

33 Zhao J, Wei N, Fan Z, et al. The mechanical properties of three types of carbon allotropes. Nanotechnology, 2013, 24: 095702

34 Song Y, Xu H, Xu B, et al. Electric current generation of a droplet falling into an electrolyte solution. Energy Convers Manage, 2020, 212: 112791

35 Zhong $\mathrm{H}, \mathrm{Li} \mathrm{X}, \mathrm{Wu} \mathrm{Z}$, et al. Two dimensional graphene nanogenerator by coulomb dragging: Moving van der Waals heterostructure. Appl Phys Lett, 2015, 106: 243903

36 Nie J, Wang Z, Ren Z, et al. Power generation from the interaction of a liquid droplet and a liquid membrane. Nat Commun, 2019, 10: 
2264

37 Adamson AW, Gast AP. Physical Chemistry of Surfaces. New York: Interscience publishers, 1967

38 Belton GR. Langmuir adsorption, the Gibbs adsorption isotherm, and interfacial kinetics in liquid metal systems. Metall Mater Trans B, 1976, 7: 35-42

39 Czepirski L, Balys MR, Komorowska-Czepirska E. Some generalization of langmuir adsorption isotherm. Internet J Chem, 2000, 3: 1099-8292

40 Loch CL, Ahn D, Chen C, et al. Sum frequency generation studies at poly(ethylene terephthalate)/silane interfaces: Hydrogen bond formation and molecular conformation determination. Langmuir, 2004, 20: 5467-5473

41 Santos EJG, Kaxiras E. Electric-field dependence of the effective dielectric constant in graphene. Nano Lett, 2013, 13: 898-902

42 Wang X, Fang S, Tan J, et al. Dynamics for droplet-based electricity generators. Nano Energy, 2021, 80: 105558

43 Wang Q, Xue M, Zhang Z. Chemical synthesis of borophene: Progress and prospective. Acta Physico-Chim Sin, 2019, 35: 565571

Acknowledgements This work was supported by the National Key Research and Development Program of China (2019YFA0705400), the National Natural Science Foundation of China (11772153 and 22073048), the Natural Science Foundation of Jiangsu Province (BK20190018), the Fundamental Research Funds for Central Universities (NJ2020003, NZ2020001, NP2019301, NJ2019002 and NC2018001) and a Project Funded by the Priority Academic Program Development of Jiangsu Higher Education Institutions.

Author contributions Zhang $\mathrm{Z}$ conceptualized this work and revised the manuscript. Zhang $\mathrm{H}$ did the calculations, derived the formulation and wrote the initial edition of the manuscript. Guo $\mathrm{W}$ revised the manuscript and offered suggestions for improving this work. All authors contributed to the general discussion.

Conflict of interest The authors declare no conflict of interest.

Supplementary information More details about derivation, electrostatic simulation and DFT calculations are available in the online version of the paper.

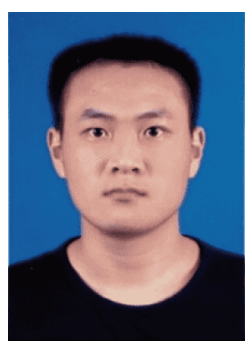

Hongbo Zhang received his BSc in engineering mechanics from Nanjing Univeristy of Aeronautics and Astronautics (NUAA). He is pursuing his $\mathrm{PhD}$ degree at NUAA under the supervision of Prof. Zhuhua Zhang and Prof. Wanlin Guo. His research focuses on the hydrovoltaic effect and the dynamic of water droplet.

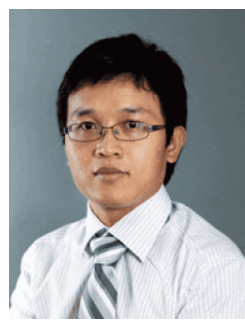

Zhuhua Zhang received his $\mathrm{PhD}$ in nanomechanics from NUAA in 2010. He has been committed to the study of nanoscale physical mechanics as well as nanoscale modeling and simulations. His research interests include structures and functionalities of low-dimensional nanomaterials, the growth mechanism of lowdimensional nanomaterials and nanostructures, and the hydrovoltaics and intelligent behaviors based on interaction between solids and liquids.

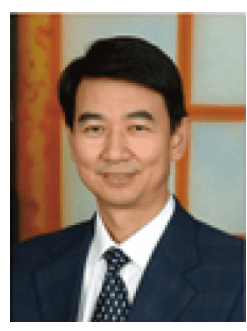

Wanlin Guo is Academician of Chinese Academy of Sciences, chair Professor in mechanics and nanoscience, founder and director of the Key Laboratory of Intelligent Nano Materials and Devices of Ministry of Education and the Institute of Nanoscience of Nanjing University of Aeronautics and Astronautics. He received the National Science Foundation of China for Distinguished Young Scholars in 1996 and the honor of Cheung Kong Scholars in 1999. His research interests include intelligent nanomaterials and devices, novel conception and technology for efficient energy conversion, hydrovoltaics and brain-like intelligence, 3D fatigue fracture and damage tolerance and durability design of structures at high temperature.

\section{在石墨烯上拖动离子液滴发电的机理}

张宏波, 张助华 ${ }^{*}$, 郭万林

摘要 最近的实验表明, 在聚合物支撑的石墨烯上移动水滴可以在 石墨烯中产生电压. 为了揭示基底、石墨烯和离子水在发电中的 作用，我们对水/石墨烯/基底界面进行了多尺度的分析和模拟，发 现被基底上表面偶极吸引到石墨烯表面的离子会驱动石墨烯中的 电荷重新分布, 从而在水/石墨烯/基底界面处形成由石墨烯中的镜 像电荷、吸附在石墨烯表面的离子以及溶液中的反离子组成的三 电层结构. 当水滴在石墨烯表面移动时, 动态形成的三电层前端动 边界驱动着石墨烯中的电荷形成电流. 采用 Langmuir 吸附理论, 结合第一性原理计算, 确定了三电层中的离子浓度, 并且估计了每 个被吸附的离子可在石墨烯中拖动的电荷量. 由此, 得到了产生的 电流与离子浓度、水滴移动速率、石墨烯厚度和基底上的偶极密 度、偶极矩之间的关系式, 且与实验测量结果吻合. 该研究结果表 明, 控制基底上的表面偶极(密度和大小)可优化水伏发电器件的性 能并促进其实际应用. 